\title{
Erratum to: Bumble bees show trait-dependent vulnerability to landscape simplification
}

\author{
Anna S. Persson ${ }^{1}$ Maj Rundlöf ${ }^{1}$ - Yann Clough ${ }^{2} \cdot$ Henrik G. Smith $^{1,2}$
}

Published online: 1 December 2015

(C) Springer Science+Business Media Dordrecht 2015

\section{Erratum to: Biodivers Conserv (2015) 24:3469-3489 DOI 10.1007/s10531-015-1008-3}

In the original publication of this article (Biodivers Conserv 24:3469-3489, 2015), we mistakenly state that males of the subgenus Bombus sensu stricto can be determined to species based on genital morphology, while in fact the determination was based on coat characteristics. This does not allow for a separation of the species B. lucorum, B. magnus and B. cryptarum with certainty. Most males (97\%) were determined to B. terrestris, indicating that this species dominated also among workers. Because these species are both morphologically similar and share categorical traits they were, as stated in the paper, combined in all analyses, except for those regarding phylogenetic signals.

The tenth line under the section, "Bumble bee and flower surveys" should read as:

Workers of species belonging to Bombus sensu stricto are not possible to separate based on morphological characters only (e.g. Williams et al. 2012b), while males may be separated based on coat characteristics. Most males $(97 \%)$ were determined to B. terrestris, indicating that this species dominated also among workers. Because these species are both morphologically similar and share categorical traits, they were combined in all analyses except for those regarding phylogenetic signals.

The online version of the original article can be found under doi:10.1007/s10531-015-1008-3.

Anna S. Persson

anna.persson@biol.lu.se

1 Department of Biology, Lund University, 22362 Lund, Sweden

2 Centre of Environmental and Climate Research, Lund University, 22362 Lund, Sweden 